\title{
Simultaneous Determination of Hydroquinone, Catechol and Resorcinol at Graphene Doped Carbon Ionic Liquid Electrode
}

\author{
Li Ma and Guang-Chao Zhao \\ Anhui key laboratory of Chemo-Biosensing, School of Chemistry and Materials Science, Anhui Normal University, \\ Wuhu 241000, China \\ Correspondence should be addressed to Guang-Chao Zhao, gczhao@mail.ahnu.edu.cn
}

Received 10 March 2012; Accepted 28 March 2012

Academic Editor: Shen-Ming Chen

Copyright ( $\odot 2012$ L. Ma and G.-C. Zhao. This is an open access article distributed under the Creative Commons Attribution License, which permits unrestricted use, distribution, and reproduction in any medium, provided the original work is properly cited.

\begin{abstract}
A new composite electrode has been prepared with doping graphene into the paste consisting graphite and ionic liquid, n-octyl-pyridinum hexafluorophosphate (OPFP). This electrode shows an excellent electrochemical activity for the redox of hydroquinone (HQ), catechol (CC), and resorcinol (RS). In comparison with bare paste electrode, the redox peaks of three isomers of dihydroxybenzene can be obviously, simultaneously observed at graphene doping paste electrode. Under the optimized condition, the simultaneous determination of HQ, CC, and RS in their ternary mixture can be carried out with a differential pulse voltammetric technique. The peak currents are linear to the concentration of HQ, CC, and RS in the range form $1 \times 10^{-5}$ to $4 \times 10^{-4}, 1 \times 10^{-5}$ to $3 \times 10^{-4}$, and $1 \times 10^{-6}$ to $1.7 \times 10^{-4} \mathrm{~mol} \mathrm{~L}^{-1}$, respectively. The limits of detection are $1.8 \times 10^{-6} \mathrm{~mol} \mathrm{~L}-1$ for $\mathrm{HQ}, 7.4 \times 10^{-7} \mathrm{~mol} \mathrm{~L}^{-1}$ for CC, and $3.6 \times 10^{-7} \mathrm{M}$ for RS, respectively.
\end{abstract}

\section{Introduction}

Catechol (CC), resorcinol (RS), and hydroquinone (HQ) are three isomers of dihydroxybenzene, which have been widely recognized as important environmental pollutants by the US Environmental Protection Agency (EPA) and the European Union (EU) due to their high toxicity and low degradability in the ecological environment [1]. The absorption of CC or HQ from the gastrointestinal tract can induce some disease such as renal tube degeneration and liver function decrease [2]. In addition, inhalation of high concentration of RS can directly lead to the death of human beings. Therefore, a highly sensitive and selective analytical method is necessary for the simultaneous determination of the three dihydroxybenzene isomers. Up to now, various methods have been exploited for the determination of the dihydroxybenzenes, such as high performance liquid chromatography (HPLC) $[3,4]$, spectrophotometry $[5,6]$ Electrochemiluminescence [7], synchronous fluorescence [8], gas chromatography/mass spectrometry [9], capillary electrochromatography [10], $\mathrm{pH}$-based-flow injection analysis [11], and electrochemical methods [12-23]. Among these methods, electrochemical method is of particular interest due to its high sensitivity, low cost, good selectivity, and rapid detection. For most electrochemical methods, more attention is focused on the simultaneous determination of CC and HQ due to their redox peaks are overlapped at ordinary electrode [12-20]. However, the simultaneous determination of HQ, $\mathrm{CC}$, and RS by electrochemical methods is few reported. Recently, a number of novel material-based electrochemical electrodes for detection of dihydroxybenzene isomers have been reported. For example, an enhanced electrochemical platform based on graphene-polyoxometalate nanomaterials for sensitive determination of diphenolic compounds had been reported [21] and the glassy carbon electrode (GCE) modified by multiwall carbon nanotubes (MWCNTs) can separate oxidation peaks of HQ and CC [12]. Moreover, the simultaneous determinations of HQ, CC, and RS have been performed at single-wall carbon nanotube (SWCNT) [22], MWCNT [23], graphene [24], graphene-chitosan [25], graphene/BMIMPF 6 composites [26], and polymer functionalized graphene [27] modified GCE. Nevertheless, it is 


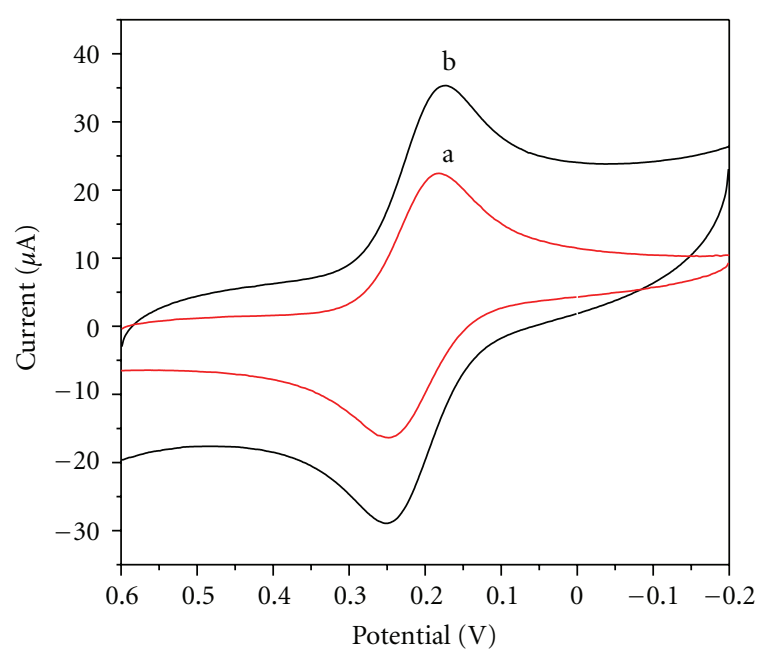

Figure 1: CVs for $0.1 \mathrm{M} \mathrm{KCl}$ solution containing $0.1 \mathrm{mM}$ $\mathrm{Fe}(\mathrm{CN})_{6}{ }^{3-/ 4-}$ at CILE (a) and graphene-doped CILE (b), scan rate: $100 \mathrm{mV} / \mathrm{s}$.

still interesting to investigate novel electrode material for the simultaneous determination of HQ, CC, and RS.

The use of solid materials, such as composite electrodes, as detectors for electrochemical analysis is commonly known [28]. Over the past five decades, carbon paste electrode as a type of composite electrode that was composed by a mixture of carbon powder and a binder has become one of the most popular electrode used as the sensors and detectors [29]. Generally, nonconductive reagents, such as mineral oil or paraffin oil were used as binders, but their electrochemical capability was poor due to their low conductivity. Therefore, it is still interesting to find a better binder to promote the performance of the carbon paste electrode. In the past years, ionic liquid (IL) has been proved to be an efficient binders and/or modifiers in the preparation of carbon composite electrodes [30]. By substituting the nonconductive organic binder with IL, carbon ionic liquid electrode (CILE) has been exhibited the advantages such as high conductivity, ease of fabrication, low cost, and renewable surface [31]. Maleki and coworkers fabricated n-octyl-pyridinium hexafluorophosphate-modified carbon paste electrode for the simultaneously determination of the dopamine, ascorbic acid, and uric acid [32]. Shang et al. reported that the direct electrochemistry of glucose oxidase (GOx) had been achieved by its direct immobilization on CILE with a conductive hydrophobic ionic liquid, 1butyl pyridinium hexafluorophosphate as binder [33], which demonstrated that CILE has its unique superiority.

In recent years, graphene, considered as a "rising star" nanostructured carbon material, is a counterpart of graphite with well-separated two-dimensional aromatic sheets composed of $\mathrm{sp}^{2}$-bonded carbon atoms. By virtue of its unique characteristic such as high surface area [34], remarkable electrical conductivity [35], and high thermal conductivity [36], graphene nanosheets have attracted more and more attentions in the preparation of sensors and biosensors. It has been reported that graphene was used as an electrode material for sensing a range of target analytes such as nitric oxide [37], dopamine [38], and hydrogen peroxide [39]. Recently, a graphene-doped carbon paste electrode was prepared with the addition of graphene into the carbon paste mixture and used for the successful determination of ascorbic acid [40]. Therefore, graphene should be an excellent modifier to fabricate carbon paste electrode.

In this paper, the graphene-doped carbon ionic liquid electrode was prepared, and the fabricated electrode can respond to all three isomers of dihydroxybenzene. Based on this, a simple and fast assay for the simultaneous determination of HQ, CC, and RS can be constructed with differential pulse voltammetry. Meanwhile, the presented method has been applied successively to simultaneous determination of $\mathrm{HQ}, \mathrm{CC}$, and RS in wastewater samples with good recovery.

\section{Experimental}

2.1. Reagents. Catechol, resorcinol, and hydroquinone were purchased from Shanghai Chemical Reagent Corporation (Shanghai, China). Graphene were purchased from Nanjing JCNANO Tech Co., Ltd. The ionic liquids, octylpyridinum hexafluorophosphate (OPFP), were purchased from Chengjie Chemical Co., Ltd. (Shanghai, China). Graphite powder (average particle size $30 \mu \mathrm{m}$ ) and other chemicals are of analytical grade. All solution was prepared by using doubly distilled water.

2.2. Apparatus. All the electrochemical experiments were carried out in a three-electrode cell controlled by $\mathrm{CHI}$ $660 \mathrm{~B}$ electrochemical workstation ( $\mathrm{CH}$ Instruments, USA). Graphene-doped CILE was used as the working electrode. An $\mathrm{Ag} / \mathrm{AgCl}$ electrode and a platinum wire electrode were used as the reference and the auxiliary electrodes, respectively. All the measurements were carried out at room temperature.

2.3. Preparation of Graphene-Doped CILE. The bare CILE was prepared by hand-mixing, in a mortar, the graphite powder and OPFP with a ratio of 50/50 (w/w). A portion of the resulting paste was packed firmly into one end of a glass tube $(\Phi=3 \mathrm{~mm})$, and a copper wire was inserted through the opposite end to establish an electrical contact. The electrode was then heated either in an oven to a temperature higher than the melting point of OPFP $\left(\mathrm{mp} 65^{\circ} \mathrm{C}\right)$ or simply by using a hair drier for $2 \mathrm{~min}$. It was then left to cool to room temperature. A new surface was obtained by smoothing the electrode onto a weighing paper. The graphene-doped CILE was fabricated using the same procedure except the introduction of a portion of graphene into the bare CILE.

\section{Results and Discussion}

3.1. Electrochemical Characteristics of the Graphene-Doped CILE. Generally, $\mathrm{Fe}(\mathrm{CN})_{6}{ }^{3-/ 4-}$ is used as a redox probe to characterize new prepared electrodes. In order to investigate the action of graphene doped into CILE, cyclic voltammetric experiments were carried out in $0.1 \mathrm{M} \mathrm{KCl}$ solution containing $0.1 \mathrm{mM} \mathrm{Fe}(\mathrm{CN})_{6}{ }^{3-/ 4-}$. Figure 1 shows the typical cyclic 

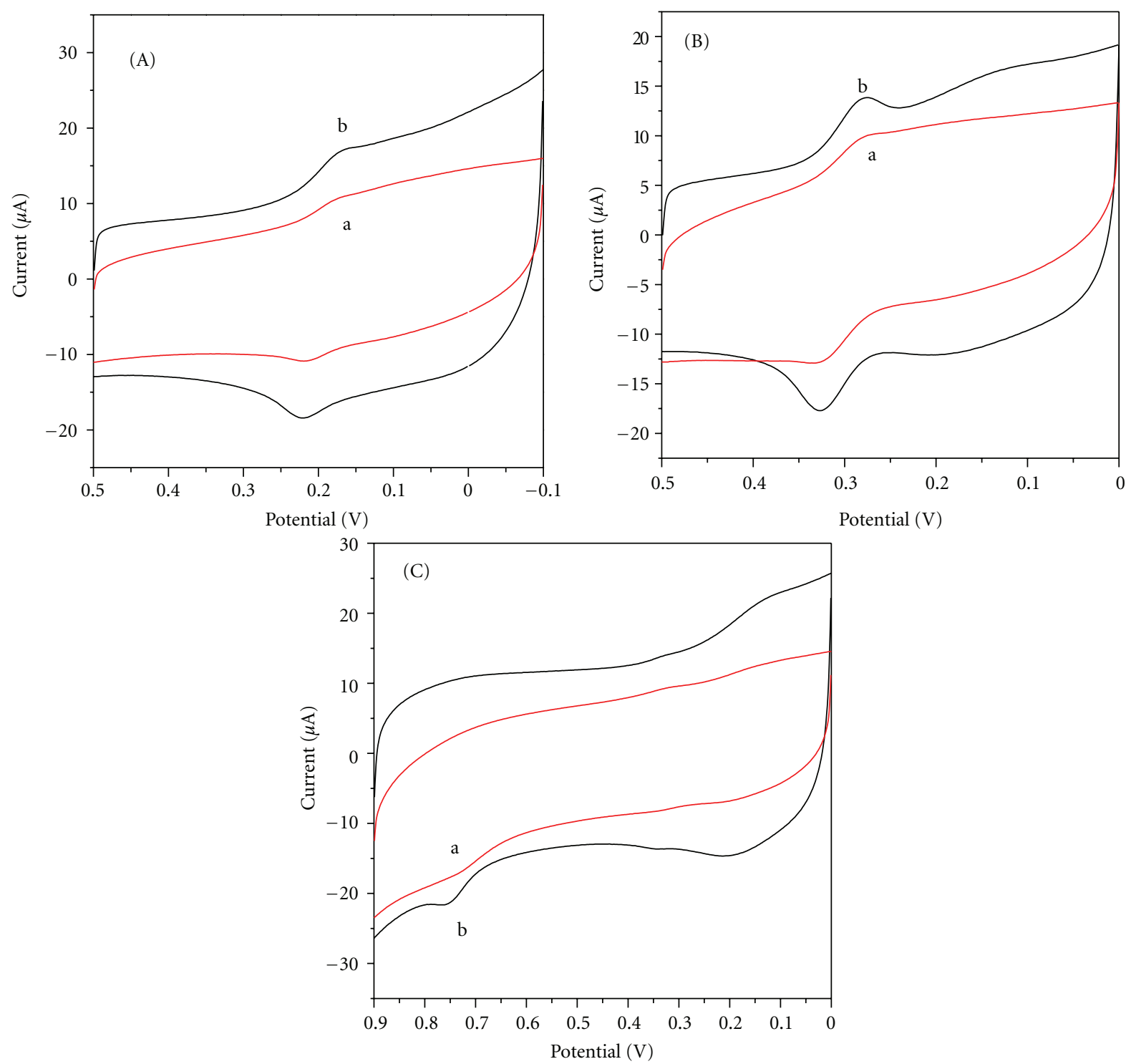

Figure 2: CVs of $5.0 \times 10^{-5} \mathrm{M}$ HQ (A), $5.0 \times 10^{-5} \mathrm{M}$ CC (B), and $1.0 \times 10^{-4} \mathrm{M}$ RS (C) in $0.10 \mathrm{M} \mathrm{HAc-NaAc}(\mathrm{pH} 5.0)$ at CILE (a) and graphene-doped CILE (b). Scan rate: $100 \mathrm{mV} / \mathrm{s}$.

voltammograms (CVs) recorded at the graphene-doped CILE and CILE, respectively. It is obvious that the electrochemical response of $\mathrm{Fe}(\mathrm{CN})_{6}{ }^{3-/ 4-}$ at graphene-doped CILE is improved, which attributes to the excellent conducting property of graphene. Meanwhile, it also indicated that the graphene was doped into the CILE successfully.

\subsection{Electrochemical Responses of HQ, CC, and RS at the Doped} CILE. In order to investigate the electrochemical response of the doped electrode to three isomers of dihydroxybenzene, $\mathrm{CV}$ experiments were carried out in acetate buffer (HAc$\mathrm{NaAc}$ ) solution containing HQ, CC, or RS, respectively. Figure 2 shows the typical cyclic voltammograms of HQ (A), CC (B), and RS (C) at the bare CILE and the doped electrode, respectively. At bare CILE, the electrochemical response of three isomers of dihydroxybenzene can be observed. But the redox peak currents are very small. Especially, for 1.0 $\times 10^{-4} \mathrm{M}$ RS, almost no anodic peak can be observed. As expected, under the same concentration, higher redox peak currents can be obtained at the graphene-doped electrode, which indicates the presence of graphene can accelerate the electron transfer between hydroquinone and electrode and the doped graphene provide a remarkable synergistic promotion for the electrochemistry of the dihydroxybenzene.

Figure 3 showed the cyclic voltammograms of the mixture of $1 \times 10^{-4} \mathrm{M} \mathrm{HQ}, \mathrm{CC}$, and RS at bare CILE and doped CILE in $0.10 \mathrm{M}$ acetate buffer (pH 5.0). At the bare CILE (curve a), two abroad, unobvious anodic peaks appear at $0.322 \mathrm{~V}$ and $0.222 \mathrm{~V}$, respectively. This is attributed to the oxidation of CC and HQ. Meanwhile, oxidation peak of RS almost cannot be observed. At the reverse scan, there are also two broad cathodic peaks appeared at about $0.259 \mathrm{~V}$ 
TABLE 1: Linear regression equations for peal potentials $\left(E_{p}\right)$ of the three isomers of dihydroxybenzene versus $\mathrm{pH}$.

\begin{tabular}{|c|c|c|c|}
\hline & HQ & $\mathrm{CC}$ & RS \\
\hline$E_{\mathrm{pa}}$ & $\begin{array}{c}E_{\mathrm{pa}}(\mathrm{V})=-0.0527 \mathrm{pH}+0.4945 \\
(R=0.9956)\end{array}$ & $\begin{array}{c}E_{\mathrm{pa}}(\mathrm{V})=-0.0542 \mathrm{pH}+0.6062 \\
(R=0.9916)\end{array}$ & $\begin{array}{c}E_{\mathrm{pa}}(\mathrm{V})=-0.0463 \mathrm{pH}+0.9951 \\
(R=0.9963)\end{array}$ \\
\hline$E_{\mathrm{pc}}$ & $\begin{array}{c}E_{\mathrm{pc}}(\mathrm{V})=-0.044 \mathrm{pH}+0.377 \\
(R=0.9956)\end{array}$ & $\begin{array}{c}E_{\mathrm{pc}}(\mathrm{V})=-0.0432 \mathrm{pH}+0.4922 \\
(R=0.9973)\end{array}$ & \\
\hline
\end{tabular}

TABLE 2: The linear regression equations for peak currents ( $I_{\mathrm{pa}}$ or $\left.I_{\mathrm{pc}}\right)$ of HQ, CC, and RS versus scan rate $(\nu)$.

\begin{tabular}{cccc}
\hline & HQ & CC & RS \\
\hline$I_{\mathrm{pa}}$ & $I_{\mathrm{pa}}=-14.73 v-3.70$ & $I_{\mathrm{pa}}=-14.18 v-6.15$ & $I_{\mathrm{pa}}=-12.2 \nu-8.79$ \\
& $(\mu \mathrm{A}, \mathrm{mV} / \mathrm{s}, R=0.9994)$ & $(\mu \mathrm{A}, \mathrm{mV} / \mathrm{s}, R=0.9990)$ & $(\mu \mathrm{A}, \mathrm{mV} / \mathrm{s}, R=0.9976)$ \\
$I_{\mathrm{pc}}$ & $I_{\mathrm{pc}}=18.99 \nu+3.409$ & $I_{\mathrm{pc}}=14.47 v+1.282$ & \\
\hline
\end{tabular}

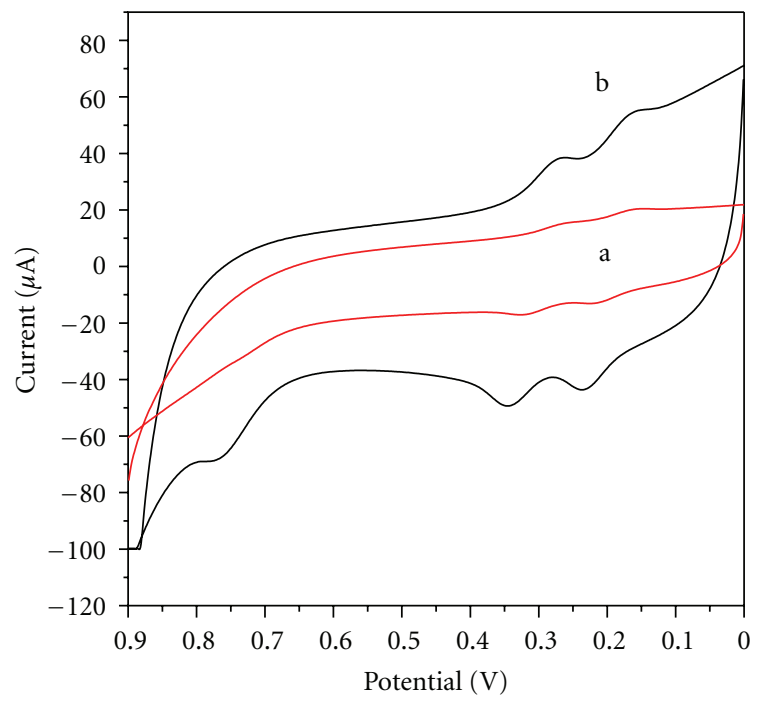

Figure 3: CVs of the mixture solution of $1 \times 10^{-4} \mathrm{M}$ CC, RS and HQ at CILE (a) and the graphene-doped CILE (b) in $0.10 \mathrm{M}$ acetate buffer (pH 5.0). Scan rate: $100 \mathrm{mV} / \mathrm{s}$.

and $0.160 \mathrm{~V}$, which correspond to the reduction peak of CC and HQ. Comparatively, at the doped CILE (curve b), three well-defined anodic peaks are observed at $0.234 \mathrm{~V}$, $0.344 \mathrm{~V}$, and $0.755 \mathrm{~V}$ for $\mathrm{HQ}, \mathrm{CC}$, and RS, respectively. In addition, two obvious cathodic peaks are obtained at 0.158 and $0.269 \mathrm{~V}$, corresponding to the reduction of the oxidation products of HQ and CC, respectively. It is clear that the anodic or cathodic peaks of CC, RS, and HQ can be distinguished effectively and all peak currents can be detected independently at the doped CILE. It is no doubt that the increase in peak currents and the decrease in the separation of the peak potential at the graphene-doped CILE can be attributed to the high surface area, excellent catalytic activity, and good conductivity of graphene. This also suggests that the simultaneous determination of three isomers of dihydroxybenzene can be performed at the graphene-doped CILE.
3.3. Electrochemical Behaviors of HQ, CC, and RS at the Doped CILE. The effect of $\mathrm{pH}$ of supporting electrolyte on the electrochemical behaviors of three isomers of dihydroxybenzene was carefully investigated in the $\mathrm{pH}$ value range from 3.0 to 7.0 and the results were shown in Figure 4. Figure 4(a) shows the relationship between the peak potential and $\mathrm{pH}$. With increasing $\mathrm{pH}$, both anodic and cathodic peak potentials decrease linearly. The linear regression equations of the three isomers of dihydroxybenzene are listed in Table 1 . The slopes of the five regression equations are close to the theory value of $58.5 \mathrm{mVpH}^{-1}$ for the electrode reaction process of equal electron and proton [42-44], indicating that the electrode process of HQ, CC, and RS at graphene-doped CILE should be a two electrons and two protons process. Figure 4(b) shows the dependence of anodic peak currents of three isomers of dihydroxybenzene on $\mathrm{pH}$. From Figure 4(b), it can be seen that the peak currents of HQ, CC, and RS increase with increasing $\mathrm{pH}$ value until $\mathrm{pH}$ reaches 5.0, and then they decrease when $\mathrm{pH}$ increases further. Therefore, considering the determination sensitivity, pH 5.0 is chosen as the optimal experimental condition.

The effect of scan rates on the CV behaviors of the three dihydroxybenzene isomers at the doped CILE is also investigated. With increasing scan rate from 50 to $500 \mathrm{mV} / \mathrm{s}$, all the redox peak currents of three dihydroxybenzene isomers increase linearly. The linear regression equations of peak currents $\left(I_{\mathrm{pa}}\right.$ or $\left.I_{\mathrm{pc}}\right)$ versus scan rate $(\nu)$ are listed in Table 2. The good linear relationship between peak currents and scan rate indicates that the electrode process of all three dihydroxybenzene isomers is a typical adsorption-controlled process.

3.4. Simultaneous Determination of HQ, CC, and RS. Differential pulse voltammetry (DPV) is performed to investigate the relationship between the peak current and concentration of three dihydroxybenzene isomers due to its higher sensitivity. The individual determination of CC, RS, or HQ in their mixtures is carried out when the concentration of one species changes whereas those of other two species remain constant. The typical cyclic voltammograms are shown Figure 5. From Figure 5(a) to 5(c), it can be seen that the determination of individual CC, HQ, or RS is independent of others. 


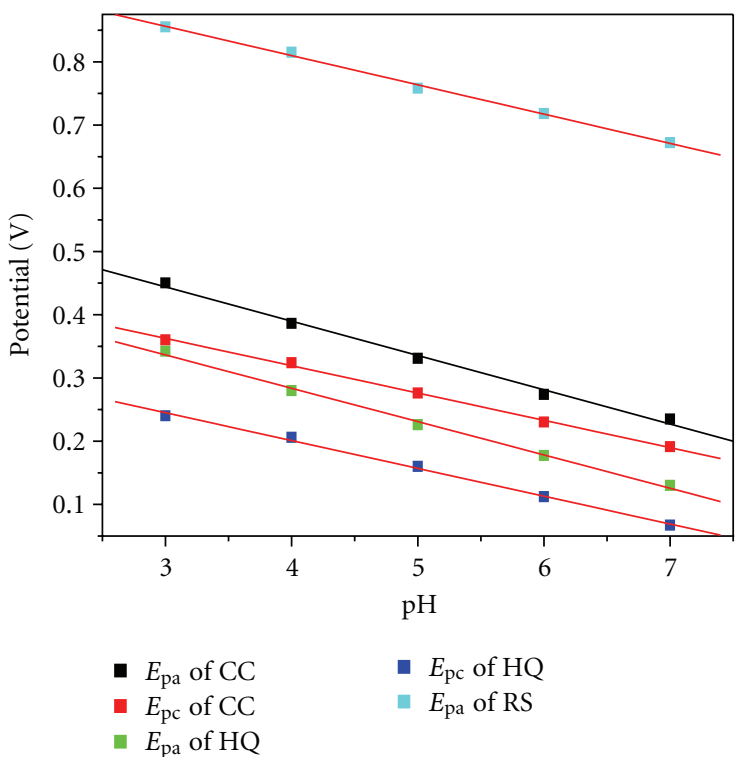

(a)

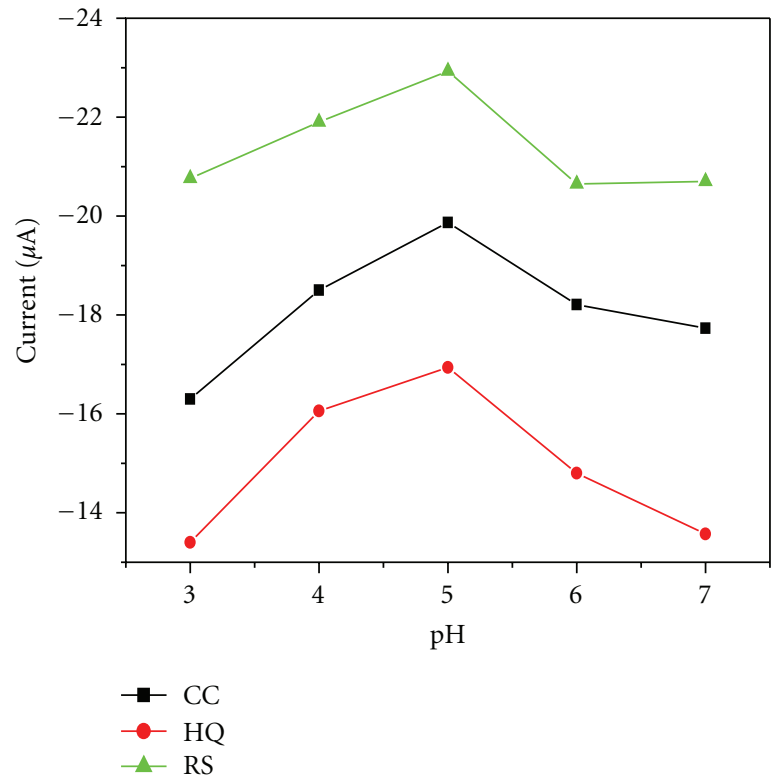

(b)

FIGURE 4: The dependences of all peak potentials (a) and anodic peak currents (b) of CC, RS, and HQ on pH.

TABLE 3: Performance comparison of the fabricated electrode for HQ, CC, and RS detection with other electrodes.

\begin{tabular}{|c|c|c|c|c|c|c|c|c|}
\hline \multirow{2}{*}{ Electrode } & \multirow{2}{*}{ Method } & \multicolumn{3}{|c|}{ Linear range $\left(10^{-6} \mathrm{M}\right)$} & \multicolumn{3}{|c|}{$\operatorname{LOD}\left(10^{-6} \mathrm{M}\right)$} & \multirow{2}{*}{ Ref. } \\
\hline & & HQ & $\mathrm{CC}$ & RS & HQ & $\mathrm{CC}$ & RS & \\
\hline MPE & DPV & $50-2000$ & $20-50$ & - & - & - & - & {$[14]$} \\
\hline IL-CPE & DPV & $10-1500$ & - & - & 4.0 & - & - & {$[15]$} \\
\hline PASA/MWNTs/GCE & DPV & $6-100$ & $6-180$ & - & 1.0 & 1.0 & - & {$[17]$} \\
\hline MWNTs/MA & Amperometry & $1-100$ & $1-100$ & $6-100$ & 0.3 & 0.2 & 0.6 & {$[41]$} \\
\hline MWNTs/GCE & DPV & $2-100$ & $2-100$ & $5-80$ & 0.6 & 0.6 & 1 & {$[23]$} \\
\hline Graphene/GCE & DPV & $1-50$ & $1-50$ & - & 0.015 & 0.010 & - & {$[24]$} \\
\hline Graphene-chitosan/GCE & DPV & $1-300$ & $1-400$ & $1-550$ & 0.75 & 0.75 & 0.75 & {$[25]$} \\
\hline Graphene/BMIMPF6/GCE & DPV & $0.5-50$ & $0.5-50$ & 一 & 0.01 & 0.02 & - & {$[26]$} \\
\hline PDDA-Graphene/GCE & DPV & $1-500$ & $1-400$ & - & 0.25 & 0.2 & - & [27] \\
\hline Graphene doped CILE & DPV & $10-400$ & $10-300$ & $1-170$ & 1.8 & 0.7 & 0.4 & This work \\
\hline
\end{tabular}

TABLE 4: Recovery results for HQ, CC, and RS in water samples.

\begin{tabular}{|c|c|c|c|c|c|c|c|c|c|c|c|c|}
\hline \multirow{2}{*}{$\begin{array}{l}\text { Sample } \\
\text { number }\end{array}$} & \multicolumn{3}{|c|}{ Detected $(\mu \mathrm{M})$} & \multicolumn{3}{|c|}{ Added $(\mu \mathrm{M})$} & \multicolumn{3}{|c|}{ Found $(\mu \mathrm{M})$} & \multicolumn{3}{|c|}{ Recovery (\%) } \\
\hline & HQ & $\mathrm{CC}$ & RS & HQ & $\mathrm{CC}$ & RS & HQ & $\mathrm{CC}$ & RS & HQ & $\mathrm{CC}$ & RS \\
\hline 1 & $\mathrm{ND}$ & $\mathrm{ND}$ & ND & 40 & 40 & 40 & 39.5 & 40.6 & 40.2 & 98.8 & 101.5 & 100.5 \\
\hline 2 & ND & ND & ND & 30 & 30 & 30 & 30.8 & 31.0 & 29.8 & 102.7 & 103.3 & 99.3 \\
\hline 3 & ND & $\mathrm{ND}$ & ND & 20 & 20 & 20 & 20.4 & 19.6 & 19.6 & 102.0 & 98.0 & 98.0 \\
\hline 4 & $\mathrm{ND}$ & ND & ND & 10 & 10 & 10 & 10.4 & 10.6 & 10.2 & 104.0 & 106.0 & 102.0 \\
\hline
\end{tabular}




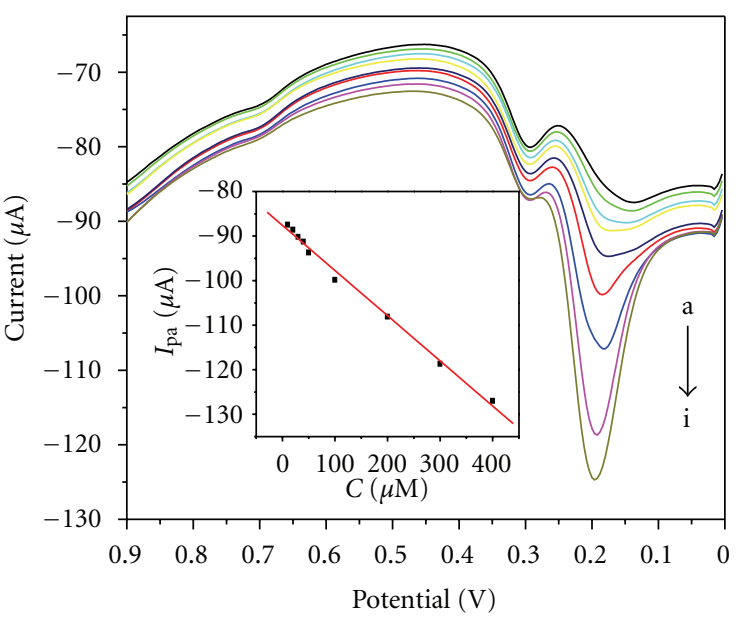

(a)

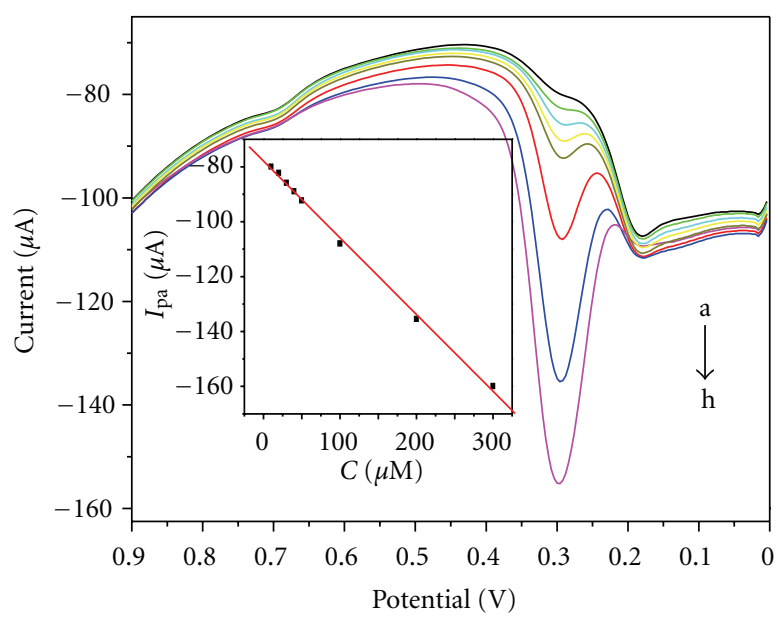

(b)

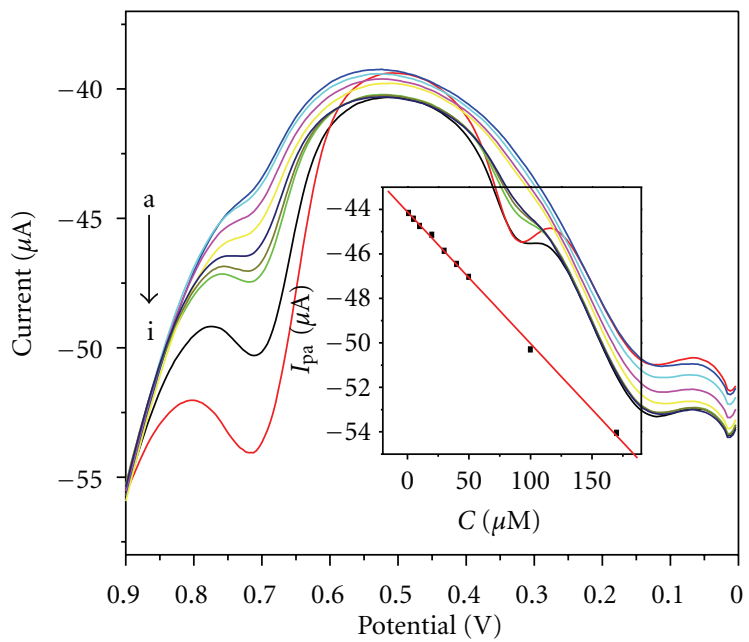

(c)

FIGURE 5: Dependences of peak currents $\left(I_{p}\right)$ on concentrations $(C)$. Inset: linear plots of $I_{p}$ versus $C$. (a) DPVs of the doped CILE in the presence of $5.0 \times 10^{-5} \mathrm{M}$ CC and RS containing different concentrations of HQ (a-i: $1.0 \times 10^{-5}, 2.0 \times 10^{-5}, 3.0 \times 10^{-5}, 4.0 \times 10^{-5}, 5.0 \times$ $10^{-5}, 1.0 \times 10^{-4}, 2.0 \times 10^{-4}, 3.0 \times 10^{-4}, 4.0 \times 10^{-4} \mathrm{M}$ ), (b) DPVs of the doped CILE in the presence of $5.0 \times 10^{-5} \mathrm{M}$ HQ and RS containing different concentrations of CC (a-h: $\left.1.0 \times 10^{-5}, 2.0 \times 10^{-5}, 3.0 \times 10^{-5}, 4.0 \times 10^{-5}, 5.0 \times 10^{-5}, 1.0 \times 10^{-4}, 2.0 \times 10^{-4}, 3.0 \times 10^{-4} \mathrm{M}\right),(\mathrm{c})$ DPVs of the doped CILE in the presence of $5.0 \times 10^{-5} \mathrm{M}$ HQ and CC containing different concentrations of RS $\left(\mathrm{a}-\mathrm{i}: 1.0 \times 10^{-6}, 5.0 \times 10^{-6}\right.$, $\left.1.0 \times 10^{-5}, 2.0 \times 10^{-5}, 3.0 \times 10^{-5}, 4.0 \times 10^{-5}, 5.0 \times 10^{-5}, 1.0 \times 10^{-4}, 1.7 \times 10^{-4} \mathrm{M}\right)$.

The good linear relationship between peak currents and the concentration of three dihydroxybenzene isomers can be obtained and is shown as inset in Figure 5. Under the optimal conditions, peak currents are linear to the concentrations in the range from $1.0 \times 10^{-5} \mathrm{M}$ to $4.0 \times 10^{-4} \mathrm{M}$ for $\mathrm{HQ}$, from $1.0 \times 10^{-5} \mathrm{M}$ to $3.0 \times 10^{-4} \mathrm{M}$ for $\mathrm{CC}$, and from 1.0 $\times 10^{-6}$ to $1.7 \times 10^{-4} \mathrm{M}$ for RS, respectively. The limit of detection (LOD) is $1.8 \times 10^{-6} \mathrm{M}$ for HQ, $7.4 \times 10^{-7} \mathrm{M}$ for $\mathrm{CC}$, and $3.6 \times 10^{-7} \mathrm{M}$ for RS, respectively. Above results suggest that this prepared electrode allows us to simultaneously and sensitively determine of CC, RS, and HQ without interference with each other. A comparison of the proposed method with other electrochemical methods is listed in Table 3. It can be seen from the table that the proposed method shows rational linear range and acceptable LOD.
3.5. Reproducibility and Stability of Graphene-Doped CILE. The reproducibility for ten doped CILEs was investigated by comparing the DPV peak current of $5 \times 10^{-5} \mathrm{M} \mathrm{HQ}, \mathrm{CC}$, and RS in a mixed solution. The relative standard deviation (RSD) is $2.53 \%$ for HQ, $2.86 \%$ for CC, and $3.70 \%$ for RS, indicating an acceptable reproducibility. Additionally, the stability of the doped electrode is also tested. The results show that the peak currents remained $94 \%$ of their initial values after the electrode was kept for 15 days.

3.6. Sample Analysis. In order to assess the possible applications of the proposed method, the simultaneous determinations of $\mathrm{HQ}, \mathrm{CC}$, and RS in local tap water samples and lake water samples were performed. However, no aimed analytes can be detected. Then, $10 \mu \mathrm{L}$ of different standard concentrations of the dihydroxybenzenes was added into 
$5.0 \mathrm{~mL}$ water sample. The amounts of $\mathrm{HQ}, \mathrm{CC}$, and RS in water samples were then determined again by calibration method, and the results are listed in Table 4 . The recoveries were in the range from $98.0 \%$ to $106.0 \%$. Therefore, the simultaneous determination of HQ, CC, and RS with the prepared electrode is feasible.

\section{Conclusions}

In this paper, a simple and sensitive electrochemical analytical method for simultaneous determination of HQ, CC, and RS has been developed employing the graphene-doped carbon ionic liquid electrode. The electrode combines the unique and attractive electrocatalytic behavior of graphene and the excellent reproducibility and good long stability of IL. The proposed electrode can be further used for multicomponent analysis in environmental control and chemical industry.

\section{References}

[1] T. Xie, Q. Liu, Y. Shi, and Q. Liu, "Simultaneous determination of positional isomers of benzenediols by capillary zone electrophoresis with square wave amperometric detection," Journal of Chromatography A, vol. 1109, no. 2, pp. 317-321, 2006.

[2] M. Lv, M. Wei, F. Rong, C. Terashima, A. Fujishima, and Z. Z. $\mathrm{Gu}$, "Electrochemical detection of catechol based on as-grown and nanograss array boron-doped diamond electrodes," Electroanalysis, vol. 22, no. 2, pp. 199-203, 2010.

[3] L.-H. Wang and Y.-P. Kuo, "Simultaneous quantitative determination of resorcinol and 1-naphthol in haircolor products by high-performance liquid chromatography," Chromatographia, vol. 49, no. 3-4, pp. 208-211, 1999.

[4] C. H. Lin, J. Y. Sheu, H. L. Wu, and Y. L. Huang, "Determination of hydroquinone in cosmetic emulsion using microdialysis sampling coupled with high-performance liquid chromatography," Journal of Pharmaceutical and Biomedical Analysis, vol. 38, no. 3, pp. 414-419, 2005.

[5] P. Nagaraja, R. A. Vasantha, and K. R. Sunitha, "A new sensitive and selective spectrophotometric method for the determination of catechol derivatives and its pharmaceutical preparations," Journal of Pharmaceutical and Biomedical Analysis, vol. 25, no. 3-4, pp. 417-424, 2001.

[6] P. Nagaraja, R. A. Vasantha, and K. R. Sunitha, "A sensitive and selective spectrophotometric estimation of catechol derivatives in pharmaceutical preparations," Talanta, vol. 55, no. 6, pp. 1039-1046, 2001.

[7] S. F. Li, X. Z. Li, J. Xu, and X. W. Wei, "Flow-injection chemiluminescence determination of polyphenols using luminol$\mathrm{NaIO}_{4}$-gold nanoparticles system," Talanta, vol. 75, no. 1, pp. 32-37, 2008.

[8] M. F. Pistonesi, M. S. Di Nezio, M. E. Centurión, M. E. Palomeque, A. G. Lista, and B. S. Fernández Band, "Determination of phenol, resorcinol and hydroquinone in air samples by synchronous fluorescence using partial leastsquares (PLS)," Talanta, vol. 69, no. 5, pp. 1265-1268, 2006.

[9] S. C. Moldoveanu and M. Kiser, "Gas chromatography/mass spectrometry versus liquid chromatography/fluorescence detection in the analysis of phenols in mainstream cigarette smoke," Journal of Chromatography A, vol. 1141, no. 1, pp. 90-97, 2007.
[10] N. Guan, Z. Zeng, Y. Wang, E. Fu, and J. Cheng, "Open tubular capillary electrochromatography in fused-silica capillaries chemically bonded with macrocyclic dioxopolyamine," Analytica Chimica Acta, vol. 418, no. 2, pp. 145-151, 2000.

[11] J. A. Garcia-Mesa and R. Mateos, "Direct automatic determination of bitterness and total phenolic compounds in virgin olive oil using a pH-based flow-injection analysis system," Journal of Agricultural and Food Chemistry, vol. 55, no. 10, pp. 3863-3868, 2007.

[12] H. Qi and C. Zhang, "Simultaneous determination of hydroquinone and catechol at a glassy carbon electrode modified with multiwall carbon nanotubes," Electroanalysis, vol. 17, no. 10, pp. 832-838, 2005.

[13] J. Peng and Z. N. Gao, "Influence of micelles on the electrochemical behaviors of catechol and hydroquinone and their simultaneous determination," Analytical and Bioanalytical Chemistry, vol. 384, no. 7-8, pp. 1525-1532, 2006.

[14] M. A. Ghanem, "Electrocatalytic activity and simultaneous determination of catechol and hydroquinone at mesoporous platinum electrode," Electrochemistry Communications, vol. 9, no. 10, pp. 2501-2506, 2007.

[15] Y. Zhang and J. B. Zheng, "Comparative investigation on electrochemical behavior of hydroquinone at carbon ionic liquid electrode, ionic liquid modified carbon paste electrode and carbon paste electrode," Electrochimica Acta, vol. 52, no. 25, pp. 7210-7216, 2007.

[16] M. Li, F. Ni, Y. Wang et al., "Sensitive and facile determination of catechol and hydroquinone simultaneously under coexistence of resorcinol with a $\mathrm{Zn} / \mathrm{Al}$ layered double hydroxide film modified glassy carbon electrode," Electroanalysis, vol. 21, no. 13, pp. 1521-1526, 2009.

[17] D. M. Zhao, X. H. Zhang, L. J. Feng, L. Jia, and S. F. Wang, "Simultaneous determination of hydroquinone and catechol at PASA/MWNTs composite film modified glassy carbon electrode," Colloids and Surfaces B, vol. 74, no. 1, pp. 317-321, 2009.

[18] Jingjing Yu, Wei Du, Faqiong Zhao, and Baizhao Zeng, "High sensitive simultaneous determination of catechol and hydroquinone at mesoporous carbon CMK-3 electrode in comparison with multi-walled carbon nanotubes and Vulcan XC-72 carbon electrodes," Electrochimica Acta, vol. 54, no. 3 , pp. 984-988, 2009.

[19] P. Yang, Q. Zhu, Y. Chen, and F. Wang, "Simultaneous determination of hydroquinone and catechol Using poly(paminobenzoic acid) modified glassy carbon electrode," Journal of Applied Polymer Science, vol. 113, no. 5, pp. 2881-2886, 2009.

[20] A. J. Saleh Ahammad, S. Sarker, M. Aminur Rahman, and J. J. Lee, "Simultaneous determination of hydroquinone and catechol at an activated glassy carbon electrode," Electroanalysis, vol. 22, no. 6, pp. 694-700, 2010.

[21] L. Cao, H. Sun, J. Li, and L. Lu, "An enhanced electrochemical platform based on graphene-polyoxometalate nanomaterials for sensitive determination of diphenolic compounds," Analytical Methods, vol. 3, no. 7, pp. 1587-1594, 2011.

[22] Z. Wang, S. Li, and Q. Lv, "Simultaneous determination of dihydroxybenzene isomers at single-wall carbon nanotube electrode," Sensors and Actuators B, vol. 127, no. 2, pp. 420425, 2007.

[23] Y. P. Ding, W. L. Liu, Q. S. Wu, and X. G. Wang, "Direct simultaneous determination of dihydroxybenzene isomers at C-nanotube-modified electrodes by derivative voltammetry," 
Journal of Electroanalytical Chemistry, vol. 575, no. 2, pp. 275280, 2005.

[24] H. Du, J. Ye, J. Zhang, X. Huang, and C. Yu, "A voltammetric sensor based on graphene-modified electrode for simultaneous determination of catechol and hydroquinone," Journal of Electroanalytical Chemistry, vol. 650, no. 2, pp. 209-213, 2011.

[25] H. Yin, Q. Zhang, Y. Zhou et al., "Electrochemical behavior of catechol, resorcinol and hydroquinone at graphene-chitosan composite film modified glassy carbon electrode and their simultaneous determination in water samples," Electrochimica Acta, vol. 56, no. 6, pp. 2748-2753, 2011.

[26] Z. Liu, Z. Wang, Y. Cao, Y. Jing, and Y. Liu, "High sensitive simultaneous determination of hydroquinone and catechol based on graphene/BMIMPF6 nanocomposite modified electrode," Sensors and Actuators B, vol. 157, no. 2, pp. 540-546, 2011.

[27] L. Wang, Y. Zhang, Y. Du, D. Lu, Y. Zhang, and C. Wang, "Simultaneous determination of catechol and hydroquinone based on poly (diallyldimethylammonium chloride) functionalized graphene-modified glassy carbon electrode," Journal of Solid State Electrochemistry, vol. 16, p. 1323, 2012.

[28] M. Luque, A. Ríos, and M. Valcárcel, "Validation of PVCgraphite composite electrodes for routine analytical work," Electroanalysis, vol. 11, no. 15, pp. 1116-1123, 1999.

[29] I. Švancara, K. Vytřas, K. Kalcher, A. Walcarius, and J. Wang, "Carbon paste electrodes in facts, numbers, and notes: a review on the occasion of the 50-years jubilee of carbon paste in electrochemistry and electroanalysis," Electroanalysis, vol. 21, no. 1, pp. 7-28, 2009.

[30] H. Liu, P. He, Z. Li et al., "An ionic liquid-type carbon paste electrode and its polyoxometalate- modified properties," Electrochemistry Communications, vol. 7, no. 12, pp. 13571363, 2005.

[31] Afsaneh Safavi, Norouz Maleki, Fariba Tajabadi, and Elahe Farjami, "High electrocatalytic effect of palladium nanoparticle arrays electrodeposited on carbon ionic liquid electrode," Electrochemistry Communications, vol. 9, no. 8, pp. 1963-1968, 2007.

[32] N. Maleki, A. Safavi, and F. Tajabadi, "High-performance carbon composite electrode based on an ionic liquid as a binder," Analytical Chemistry, vol. 78, no. 11, pp. 3820-3826, 2006.

[33] X. Shang, H. Zhang, and J. Zheng, "Direct electrochemistry of glucose oxidase immobilized on NdPO4 nanoparticles/chitosan composite film on glassy carbon electrodes and its biosensing application," Electrochemistry Communications, vol. 10, pp. 1140-1143, 2008.

[34] M. D. Stoller, S. Park, Z. Yanwu, J. An, and R. S. Ruoff, "Graphene-Based ultracapacitors," Nano Letters, vol. 8, no. 10, pp. 3498-3502, 2008.

[35] K. I. Bolotin, K. J. Sikes, Z. Jiang et al., "Ultrahigh electron mobility in suspended graphene," Solid State Communications, vol. 146, no. 9-10, pp. 351-355, 2008.

[36] A. A. Balandin, S. Ghosh, W. Bao et al., "Superior thermal conductivity of single-layer graphene," Nano Letters, vol. 8, no. 3, pp. 902-907, 2008.

[37] J. F. Wu, M. Q. Xu, and G. C. Zhao, "Graphene-based modified electrode for the direct electron transfer of Cytochrome $\mathrm{c}$ and biosensing," Electrochemistry Communications, vol. 12, no. 1, pp. 175-177, 2010.

[38] Y. R. Kim, S. Bong, Y. J. Kang et al., "Electrochemical detection of dopamine in the presence of ascorbic acid using graphene modified electrodes," Biosensors and Bioelectronics, vol. 25, no. 10, pp. 2366-2369, 2010.

[39] K. Zhou, Y. Zhu, X. Yang, J. Luo, C. Li, and S. Luan, "A novel hydrogen peroxide biosensor based on Au-grapheneHRP-chitosan biocomposites," Electrochimica Acta, vol. 55, no. 9, pp. 3055-3060, 2010.

[40] F. Li, J. Li, Y. Feng, L. Yang, and Z. Du, "Electrochemical behavior of graphene doped carbon paste electrode and its application for sensitive determination of ascorbic acid," Sensors and Actuators B, vol. 157, no. 1, pp. 110-114, 2011.

[41] D. Zhang, Y. Peng, H. Qi, Q. Gao, and C. Zhang, "Application of multielectrode array modified with carbon nanotubes to simultaneous amperometric determination of dihydroxybenzene isomers," Sensors and Actuators B, vol. 136, no. 1, pp. 113121, 2009.

[42] W. Sun, M. Xi, L. Zhang, T. Zhan, H. Gao, and K. Jiao, "Electrochemical behaviors of thymine on a new ionic liquid modified carbon electrode and its detection," Electrochimica Acta, vol. 56, no. 1, pp. 222-226, 2010.

[43] P. Wu, Q. Shao, Y. Hu et al., "Direct electrochemistry of glucose oxidase assembled on graphene and application to glucose detection," Electrochimica Acta, vol. 55, no. 28, pp. 8606-8614, 2010.

[44] B. J. Sanghavi and A. K. Srivastava, "Simultaneous voltammetric determination of acetaminophen, aspirin and caffeine using an in situ surfactant-modified multiwalled carbon nanotube paste electrode," Electrochimica Acta, vol. 55, no. 28, pp. 8638-8648, 2010. 


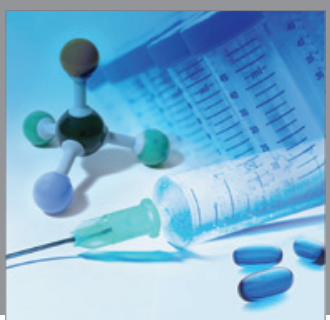

International Journal of

Medicinal Chemistry

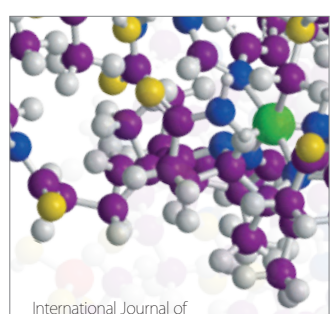

Carbohydrate Chemistry

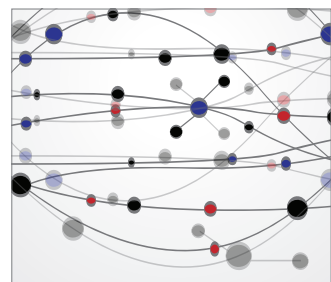

The Scientific World Journal
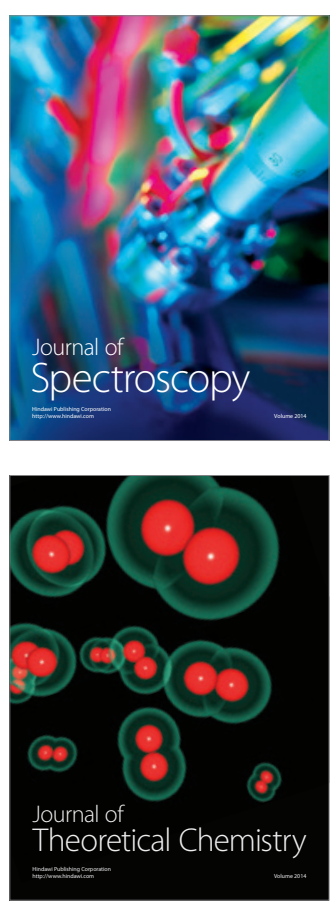
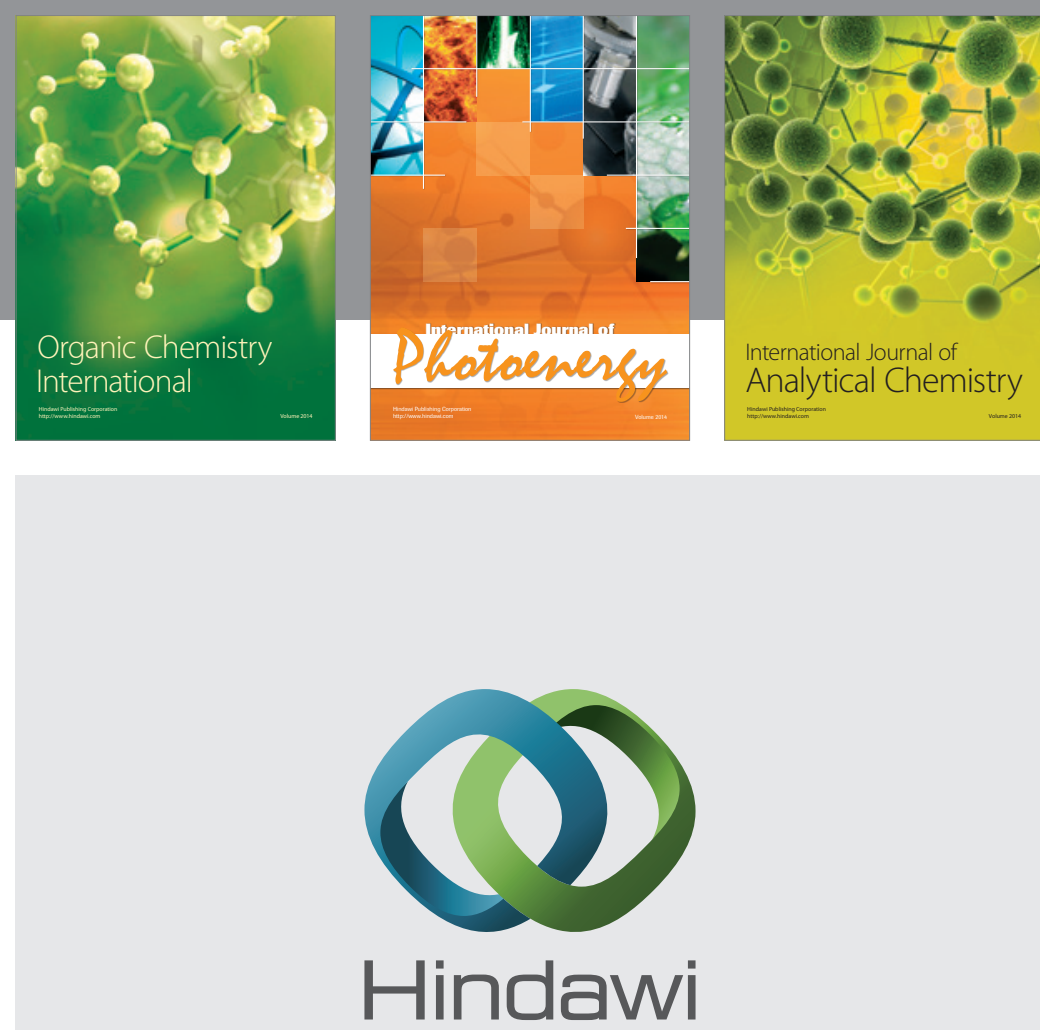

Submit your manuscripts at

http://www.hindawi.com
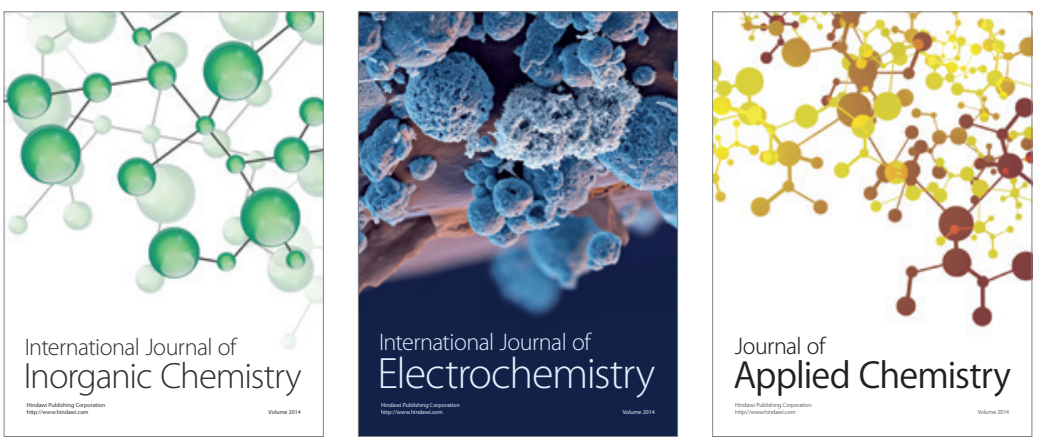

Journal of

Applied Chemistry
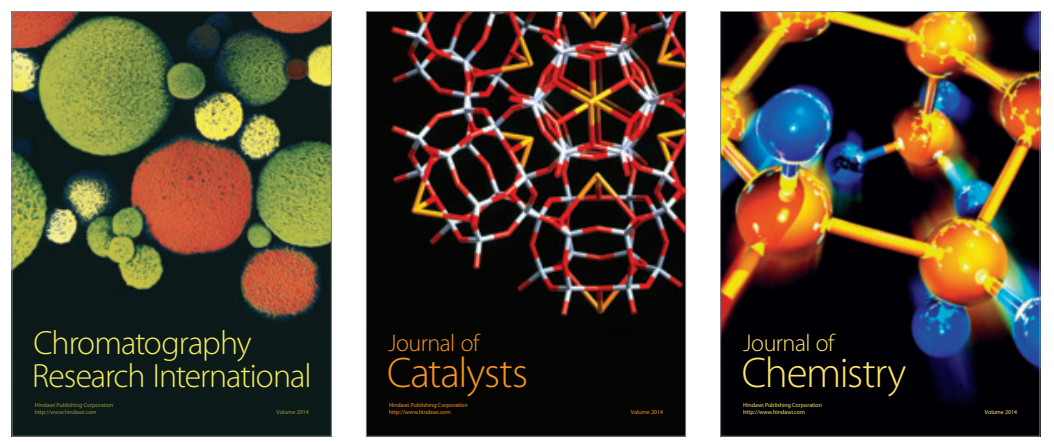
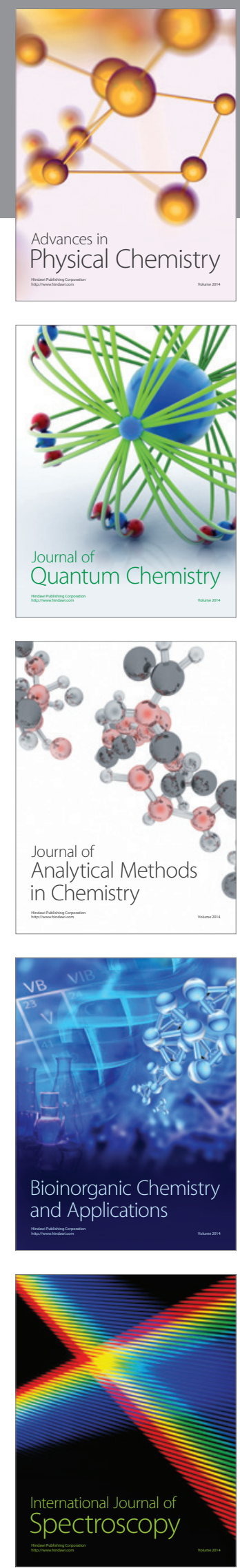